\title{
HDC: A subroutine library for image processing using Burt's hierarchical discrete correlation
}

\author{
MIGUEL A. GARCÍA-PÉREZ \\ Universidad Complutense, Madrid, Spain
}

\begin{abstract}
This paper describes a library of subroutines that efficiently implement Burt's (1981) hierarchical discrete correlation (HDC). The subroutines are written in Professional FORTRAN for IBM PCs or compatibles. Subroutines are included for both even and odd forms of the HDC, as well as for the corresponding algorithms for pyramid processing. Odd-width kernels of up to $7 \times 7$ pixels and even-width kernels of up to $6 \times 6$ pixels can be programmed easily with these subroutines. Thus, this library contains the forms of HDC that are most widely used in image processing and visual modeling. I show the efficiency of this implementation by reporting the execution time of each subroutine for several image sizes. I also present a method that simulates rotation of the kernel in order to allow applying kernels without circular symmetry at the desired orientation.
\end{abstract}

Filtering is one of the most widely used methods in digital image processing, and it is also a key concept in current models of human visual information processing. Assuming space-invariance, linear filtering operations can be described as convolutions of the input image with the kernel (the impulse response) that characterizes the filter under consideration. For practical applications on serial computers, this kind of linear filtering operation is usually performed using the detours through the frequency domain, which are justified by the well-known convolution theorem (Bracewell, 1978). Under that approach, the output image is computed as the inverse Fourier transform of the product of the direct Fourier transforms of the input image and the filter impulse response. Fast Fourier transform (FFT) algorithms make faster this otherwise long and indirect method, and new FFT algorithms are being developed continually to reduce the number of operations (and, therefore, the computer time) needed to perform these computations (see, e.g., Duhamel, 1986).

Beside this work on FFT algorithms, some attention also has been directed to computational schemes that result in a more efficient pattern than either direct convolution or convolution via FFT. This is the case, for example, with Burt's (1981) hierarchical discrete correlation (HDC) and with Crowley and Stern's (1984) difference of low-pass transform (DOLP). Both techniques yield the same results as does a brute force procedure, but at considerably less computational expense.

The principle underlying the HDC is cascaded filtering. In the linear, space-invariant case, the operation of

This work was carried out at the Laboratorio de Vision of the Facultad de Psicología, Universidad Complutense, with financial support from the Fundación Ramón Areces for the research project Visión Artificial y Visión Humana: Aplicaciones a la Percepción Visual en Robótica. I thank P. J. Burt for helpful comments on his hierarchical discrete correlation. Address correspondence to Miguel A. García-Pérez, Laboratorio de Visión, Facultad de Psicología, Universidad Complutense, Campus de Somosaguas, 28023 Madrid, Spain. a set of $n$ cascaded filters, each with impulse response $h_{i}$, is equivalent to the operation of a single filter whose impulse response is equal to the convolution of the impulse responses of all the cascaded filters, and, inversely, any filter can be decomposed into a set of cascaded filters, each with a suitably chosen impulse response. For digital filters (therefore having a finite spatial spread), the results of convolving an image with a large kernel can also be obtained by performing recursively (i.e., in cascade) a convolution with smaller kernels. This process has at least two interesting by-products. First, the number of operations (additions and multiplications) required for each pixel decreases significantly: a $9 \times 9$ kernel applied directly requires 81 operations per pixel, whereas the use of two $5 \times 5$ kernels in cascade yields the same result with only 50 operations per pixel ( 25 at each step). Second, the images obtained at each step of the process may have interesting properties. Also, since filtering operations result in a bandwidth reduction, the output at each step can be subsampled without loss of information and with the additional advantage of reducing the computational load from step to step.

All these ideas are nicely and efficiently combined in the HDC. The kernel, $w$, of the HDC can indistinctly have even or odd width, but it is subjected to certain constraints to ensure that the filtered images have particular properties (Burt, 1981, 1983). If the kernel of the convolution has width $k(k>1),{ }^{1}$ then these constraints imply that the number of free parameters in that kernel is equal to $\hat{m}-1$, where $\hat{m}=k / 2$ for even $k$ and $\hat{m}=(k-1) / 2$ for odd $k$. Consequently, the range of different filter shapes available for the equivalent weighting function (see Burt, 1981) depends on the width of the generating kernel.

The HDC is being used increasingly in image analysis, computer vision, and human visual modeling (see e.g., Burt, 1983; Burt \& Adelson, 1983a, 1983b; Burton, Haig, \& Moorhead, 1986; García-Pérez, 1986; Graham, Kramer, \& Haber, 1985; Richards, Dawson, \& Whitting- 
ton, 1986), and a processor system has been designed to perform HDC computations on NTSC video images at a 30-frames-per-second real-time rate (Arbeiter \& Bessler, 1986). The purpose of this paper is to describe a library of subroutines that perform the most basic and widely used HDC and related pyramid operations. The next section describes the general aspects of the library. Then the algorithm implemented by each subroutine is described, along with its calling sequence, and some images are shown that exemplify the results of their application.

\section{General Description of the Library}

This library source code is written in Professional FORTRAN (Ryan-McFarland, 1984) for IBM PCs or compatibles. All subroutines reside in a library of object modules, named HDC, and they can therefore be referenced from any user-made main program written in this language.

The input image passed to the subroutines should be stored in a two-dimensional short-integer array (INTEGER $* 2$ in Professional FORTRAN). Although all computations are performed in single-precision reals (REAL*4 in Professional FORTRAN), the final results are rounded to the nearest integer so that the output image returned by the subroutines is also stored in a short-integer array. This makes efficient use of computer storage: $256 \times 256 \mathrm{im}$ ages with up to 16 bits per pixel can be handled easily on a computer with $640 \mathrm{~K}$.

Although Burt (1981; see also Burt \& Adelson, 1983a) considered image arrays with their lower limits set at 0 and their upper limits at $n-1$ ( $n$ being the size of the image), the DIMENSION statements in our subroutines have been written using FORTRAN IV standards (i.e., specifying $n$ as the dimension of the array and implicitly taking 1 as its lower limit) for purposes of consistency with the rest of our software. Nonetheless, it would cause no problems to users who dimension arrays in their main programs as Burt suggested, since the length of the arrays passed to the subroutines is independent of the particular method used in dimensioning them. It is important to note, however, that the definitions of HDC algorithms should be adapted to this circumstance, and that is the reason why Equations 1-6 presented in the following section differ in some respects from Burt's otherwise equivalent equations.

An important aspect in image processing is the treatment of image boundaries. Even though only local computations were involved (as in the HDC), the application of any algorithm would require in some cases the use of image points that fall outside the edges of the image under consideration. The treatment of image boundaries (i.e., what to do in these cases) is not a trivial issue, and some important aspects of the output depend on the choice for this treatment. Each of the subroutines in this library can be directed to treat image boundaries in any of four different ways, which are described next. Let $f$ be a onedimensional image containing $n$ pixels. If, in order to compute the value at any point in the output image $g$, an image point is required whose location, $i$, in the input image does not satisfy $1 \leq i \leq n$, then any of the following rules can be used:

Rule a. Zeros outside the image edge. This is the noncyclic convolution implemented in many image processing subroutines. The rule that applies here is as follows:

$$
\begin{array}{ll}
f(i)=0 & \text { if } i<1 \\
f(i)=0 & \text { if } i>n .
\end{array}
$$

Rule b. Periodicity of the image. This method assumes that the input image represents a single period of a periodic function. Therefore, the image is merely replicated at either of its edges in order to get the required points. Application of this method was described by Watson and Ahumada (1985). The rule that describes this instance of boundary treatment is the following:

$$
\begin{array}{ll}
f(i)=f(i+n) & \text { if } i<1 \\
f(i)=f(i-n) & \text { if } i>n .
\end{array}
$$

Rule c. Reflection along image boundaries. This method was described by Burt (1983), and the corresponding rule is as follows:

$$
\begin{array}{ll}
f(i)=f(2-i) & \text { if } i<1 \\
f(i)=f(2 n-i) & \text { if } i>n .
\end{array}
$$

Rule $d$. Reflection and inversion along image boundaries. This method was discussed by Burt and Adelson (1983b), and it is of particular importance for the application described in that paper. The corresponding rule is

$$
\begin{array}{ll}
f(i)=2 f(1)-f(2-i) & \text { if } i<1 \\
f(i)=2 f(n)-f(2 n-i) & \text { if } i>n .
\end{array}
$$

All four boundary conditions are illustrated in one dimension in Figure 1. For two-dimensional images, the selected method is applied equally and independently to rows and columns.

\section{Subroutines Description}

Sixteen basic subroutines are included in this library. ${ }^{2}$ They implement the even and odd forms of the HDC algorithm, as well as of the REDUCE and EXPAND functions (see Burt \& Adelson, 1983a) required for pyramid processing. Each subroutine has a six-character name, in which the first three characters refer to the type of operation the subroutine performs (HDC or SMH for HDC subroutines, RED for REDUCE subroutines, or EXP for EXPAND subroutines), the fourth character refers to the form of the algorithm (either $E$ for even or $O$ for odd), the fifth character refers to the type of arrays (I for integer in this case), and the last character is a number that refers to the lateral spread, $\hat{m}$, of the generating kernel (either 2 or 3 in these subroutines, and, therefore, the width of the generating kernel is $k=2 \hat{m}+1=5$ or 7 for the odd form, and $k=2 \hat{m}=4$ or 6 for the even form 


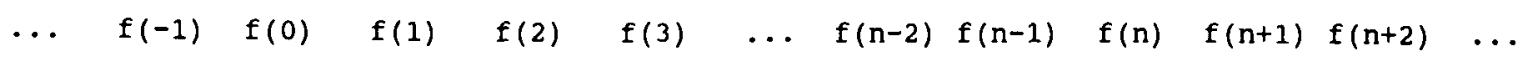

\begin{tabular}{|l|l|l|l|l|l|l|}
\hline 110 & 115 & 120 & $\ldots$ & 52 & 70 & 88 \\
\hline
\end{tabular}

(a)

\begin{tabular}{ccc|c|c|c|c|c|c|c|c|c|c|c|c|}
\hline$\ldots$ & 0 & 0 & 110 & 115 & 120 & $\ldots$ & 52 & 70 & 88 & 0 & 0 \\
\hline
\end{tabular}

(b)

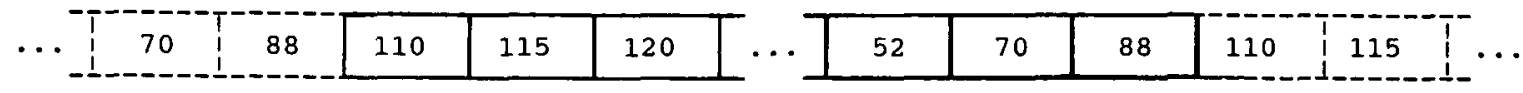

\begin{tabular}{ll|l|l|l|l|}
\hline & 120 & 115 & 110 & 115 & 120 \\
\hline
\end{tabular}

\begin{tabular}{|l|l|}
\hline 52 & \\
\hline
\end{tabular}

70

$88\left[\begin{array}{c|c|c}70 & 52 & \ldots\end{array}\right.$

(d)

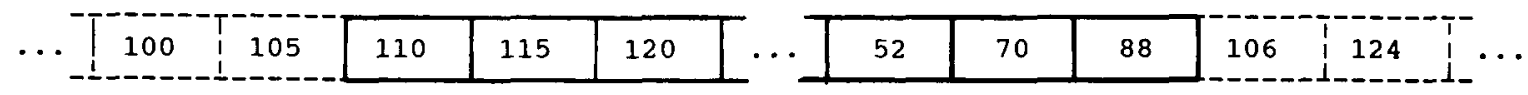

Figure 1. Application of the different methods to treat image boundaries. The top row shows arbitrary values at the three nearest points to either edge of a one-dimensional image of size $n$. The rows labeled (a) through (d) show the values that would be assigned by the corresponding rules described in the text to the two nearest image points falling outside either edge of the image.

of each algorithm). The order, $r$, of the convolution is $r=1$ for HDC subroutines (whose operation proceeds, therefore, without subsampling) and $r=2$ for both REDUCE and EXPAND subroutines (which, therefore, proceed by subsampling every other pixel from level to level in either dimension in order to construct the pyramid). Although both even and odd forms of the hierarchical filtering can be performed on images of any size, We follow Burt (personal communication, October 1986) and consider from here on that the odd form is applied to images of odd width, whereas the even form is applied to images of even size. (This point is important only for pyramid operations, however.)

Next, we describe the algorithm each subroutine implements and its calling sequence.

Subroutines HDCOI2 and SMHOI2 (HDC algorithm, odd form, integer arrays, $\hat{\boldsymbol{m}}=2$ ). Let $f(i, j)$ be the input image to the subroutine, where $1 \leq i \leq R$, $1 \leq j \leq C$, and $R$ and $C$, respectively, represent the total number of rows and columns in the image array. The output image, $g(i, j)$, is computed as

$$
g(i, j)=\sum_{m, n=-2}^{2} \sum(m, n) f(i+m, j+n),
$$

where $w(m, n)$ is the generating kernel. For computational convenience (see Burt, 1981), this kernel is constrained to be separable [i.e., $w(m, n)=w_{x}(n) w_{y}(m)$ ] and symmetrical [i.e., $w_{x}(-2)=w_{x}(2)$ and $w_{x}(-1)=w_{x}(1)$, and similarly for $w_{y}$ ]. Also let $w_{x}(0)=a_{x}, w_{x}(1)=b_{x}, w_{x}(2)=$ $c_{x}, w_{y}(0)=a_{y}, w_{y}(1)=b_{y}$, and $w_{y}(2)=c_{y}$ (Burt, 1981). Then an additional constraint (equal contribution; see Burt, 1981) implies that only the central weight parameters $a_{x}$ (for rows) and $a_{y}$ (for columns) need to be specified.
Burt (1981) also imposed each one-dimensional kernel to be normalized; that is,

$$
\sum_{n=-2}^{2} w_{x}(n)=\sum_{m=-2}^{2} w_{y}(m)=1 .
$$

This constraint makes kernels act as lowpass filters insofar as the output corresponding to a uniform input is uniform and with the same amplitude as the input, and it happens even if the kernel has any negative weight (as in Burt \& Adelson, 1983a, top of Figure 3). In order to allow the design of strict bandpass kernels that yield null response to uniform input, we relax this constraint, letting

$$
\sum_{n=-2}^{2} w_{x}(n)=s_{x}
$$

and

$$
\sum_{m=-2}^{2} w_{y}(m)=s_{y},
$$

which includes Burt's constraint of normalization as a particular case. In general, $s_{x}$ and $s_{y}$ may have any value, but only the value 0 differs qualitatively from the other values. All nonzero values give rise to kernels that have a normalized equivalent kernel save for a scaling factor. Parameters $s_{x}$ and $s_{y}$ can, therefore, be used to control the gain of the kernel.

Subroutine HDCOI2 should be used when the image is more than three columns wide. If $C \leq 3$, then subroutine SMHOI2 should be used instead. The calling sequences of these subroutines are 


\section{CALL HDCOI2 (F,G,WK,NR,NC,AX,SX,AY, SY,BT)}

and

CALL SMHOI2 (F,G,WK,NR,NC,AX,SX,AY, SY,BT)

where

F: two-dimensional array of dimensions $\mathrm{NR} \times \mathrm{NC}$ containing the input image (INTEGER $* 2$ ).

G: two-dimensional array of the same size as $F$ that is to contain the output image (INTEGER $* 2$ ).

WK: work vector of at least length NR $(R E A L * 4)$.

NR: number of rows in both $F$ and $G$ arrays (INTEGER*4).

NC: number of columns in both $F$ and $G$ arrays (INTEGER*4).

AX: central weight parameter of the generating kernel in the $x$-(row)direction (REAL*4).

SX: sum of weights for the kernel in the $x$-direction (REAL*4).

AY: central weight parameter of the generating kernel in the $y$-(column)direction (REAL $* 4$ ).

$S Y$ : sum of weights for the kernel in the $y$-direction (REAL*4).

BT: option for boundary treatment (INTEGER*4):

$$
\begin{aligned}
& \mathrm{BT}=0 \longleftrightarrow \text { rule a above, } \\
& \mathrm{BT}=1 \longleftrightarrow \text { rule } \mathrm{b} \text { above, } \\
& \mathrm{BT}=2 \longleftrightarrow \text { rule c above, } \\
& \mathrm{BT}=3 \longleftrightarrow \text { rule } \mathrm{d} \text { above },
\end{aligned}
$$

Subroutine HDCEI2 and SMHEI2 (HDC algorithm, even form, integer arrays, $\hat{\boldsymbol{m}}=2)$. These subroutines implement the same algorithm as do the previous ones, after adaptation to the even width of the generating kernel. However, that generating kernel cannot be centered on the pixels of the input image due to its even width; therefore, it has to be displaced either to the left or to the right (in one dimension) in order to calculate the values at every point in the output image. Thus, the output image can be obtained using any of the following algorithms (in one dimension):

$$
g(i)=\sum_{m=-2}^{1} w_{x}(m) f(i+m)
$$

or

$$
g(i)=\sum_{m=-1}^{2} w_{x}(m) f(i+m)
$$

Obviously, there are four possible permutations of the two algorithms when dealing with two-dimensional images. These definitions are slightly different from Burt's (1981) in that all array indexes are integers instead of halfintegers. Both definitions are otherwise equivalent. Ker- nels of even width are subjected to the same constraints as are their odd counterparts. Therefore, $w_{x}(-2)=w_{x}(1)$ $=b_{x}$ and $w_{x}(-1)=w_{x}(0)=a_{x}$ (for the algorithm in Equation 2.1), or $w_{x}(-1)=w_{x}(2)=b_{x}$ and $w_{x}(0)=w_{x}(1)=$ $a_{x}$ (for that in Equation 2.2), and similarly for $w_{y}$. As it happens with the odd form of the algorithm, only the central weight parameter and the sum of weights in each direction need to be specified.

If $C<3$, then subroutine SMHEI 2 should be used instead of subroutine HDCEI2. These subroutines are called by using the statements

\section{CALL HDCEI2 (F,G,WK,NR,NC,AX,SX,AY,SY, BT,ALGX,ALGY)}

or

\section{CALL SMHEI2 (F,G,WK,NR,NC,AX,SX,AY,SY, BT,ALGX,ALGY)}

where the types and definitions of the first 10 arguments are the same as for the previous subroutines, and

ALGX: form of the algorithm in the $x$-direction (INTEGER*4):

ALGX $=1 \longleftrightarrow$ algorithm represented in Equation 2.1,

ALGX $=2 \longleftrightarrow$ algorithm represented in Equation 2.2, and similarly for ALGY but applied to the vertical filter. Neither of the algorithms seems to have any particular advantage over the other, and we recommend alternating them when the subroutine is to be used recursively so that the image does not get systematically displaced from level to level due to the displacement of the generating kernel (see Figure 2).

Subroutine REDO12 (REDUCE function, odd form, integer arrays, $\hat{m}=2$ ). In the previous subroutines, the order of the filtering was always $r=1$. In this one, the order becomes $r=2$, and it remains the same for the next three subroutines. Therefore, the size of the output image is different from that of the input, which is particularly useful for pyramid operations. Actually, for odd pyramid operations, the width of the output image is roughly half the width of the input array. Let $g_{l}(i, j)$ be the image in level $l$ of the pyramid, which contains $C_{t}$ columns and $R_{l}$ rows of pixels. Then, the next level in the pyramid is computed by this subroutine as

$$
g_{l+1}(i, j)=\sum_{m, n=-2}^{2} \sum w(m, n) g_{1}(2 i+m-1,2 j+n-1)
$$

where $1 \leq i \leq R_{l+1}, 1 \leq j \leq C_{l+1}, R_{l+1}=\left(R_{l}+1\right) / 2$, and $C_{l+1}=\left(C_{t}+1\right) / 2$. The dimensions of the original image (level 0 in the pyramid) are appropriate for odd pyramid construction (Burt \& Adelson, 1983a) if integers $M_{C}$ and $M_{R}\left(M_{C}\right.$ or $M_{R}$ or both must be odd) and integer $N$ exist such that $C_{0}=M_{C} 2^{N}+1$ and $R_{0}=M_{R} 2^{N}+1$. This implies that the odd REDUCE function can be applied to any image in which the number of pixels per side is odd, although the number of possible levels in the pyramid depends on that particular value. In fact, $N$ levels can be 


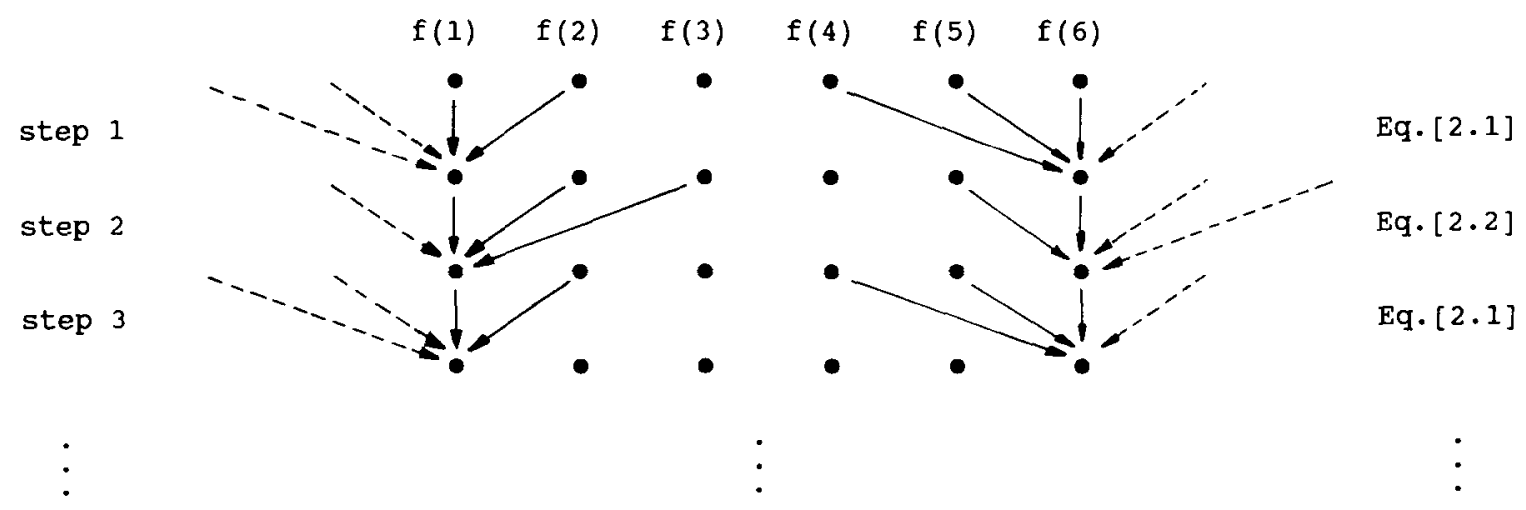

Figure 2. The top row shows the dots that constitute a one-dimensional image of width 6 , which is the starting point of a recursive application of the even-HDC algorithm. The rows below that show the images obtained by that recursive process. One of the two possible forms of alternation between the algorithms represented in Equations 2.1 and 2.2 to avoid systematic displacement of the image from step to step is shown on the right-hand side. Arrows indicate (only for edge pixels) which points in a given image contribute to the value at a point in the next image, depending on the choice for the algorithm.

constructed, or, put another way, the recursive process stops the first time the output image has an even number of pixels per side. Thus, if the original image has width $257\left(C_{0}=R_{0}=257, M_{C}=M_{R}=1, N=8\right)$, then eight more images can be added to the pyramid whose widths will be $129,65,33,17,9,5,3$, and 2 pixels, respectively. The calling sequence of this subroutine is

\section{CALL REDOI2 (F,G,WK,NRF,NCF,NRG,NCG, AX,SX,AY,SY,BT)}

where

F: two-dimensional array of dimensions NRF $\times$ NCF containing the input image in level $l$ of the pyramid (INTEGER $* 2$ ).

G: two-dimensional array of dimensions NRG $\times$ NCG, which is to contain the output image in level $l+1$ (INTEGER*2).

WK: work vector of at least length NRF (REAL*4). NRF: number of rows in array F (INTEGER*4).

NCF: number of columns in array $F$ (INTEGER*4).

NRG: number of rows in array $G$ [NRG $=(N R F+1) / 2]$ (INTEGER*4).

NCG: number of columns in array $G[N C G=$ $(\mathrm{NCF}+1) / 2$ ] (INTEGER *4).

AX, SX, AY, SY, and BT are the same as in the previous subroutines.

Subroutine REDEI2 (REDUCE function, even form, integer arrays, $\hat{m}=2$ ). The even form of the REDUCE function differs in no significant respect from its odd form, except in how the even size of the arrays affects the calculations. Therefore, all the points raised in the discussion of the previous subroutine can be applied directly to this one by merely replacing "even" with "odd," and vice versa. Thus, each level in the pyramid is exactly half the size of its predecessor, and the constraints on the size of the original image for even pyramid processing become $C_{0}=M_{C} 2^{N}$ and $R_{0}=M_{R} 2^{N}$, and, therefore, the size of each successive level in the pyramid representation of a $256 \times 256$ image is $128,64,32,16,8,4,2$, and 1 pixels per side. Displacement of the even-width generating kernel is no longer necessary when performing pyramid operations, since image points in the output do not correspond in position with points at the input image, as shown in Figure 3 in one dimension. Each level in the pyramid is obtained from the previous one using

$$
g_{l+1}(i, j)=\sum_{m, n=-2}^{1} \sum_{i} w(m, n) g_{l}(2 i+m, 2 j+n),
$$

where $1 \leq i \leq R_{l+1}, 1 \leq j \leq C_{l+1}, R_{l+1}=R_{l} / 2$, and $C_{l+1}=C_{t} / 2$. The calling sequence of the subroutine is

\section{CALL REDEI2 (F,G,WK,NRF,NCF,NRG,NCG,AX, SX,AY,SY,BT)}

where the definitions and types of the arguments are the same as for the previous subroutine, except that now $\mathrm{NRG}=\mathrm{NRF} / 2$ and $\mathrm{NCG}=\mathrm{NCF} / 2$.

Subroutine EXPO12 (EXPAND function, odd form, integer arrays, $\hat{\boldsymbol{m}}=2)$. Let $g_{\imath, n}(i, j)$ be the result of expanding the image in level $l$ of the pyramid to the size of the image in level $l-n$. As a matter of course, $g_{l, 0}(i, j)$ $=g_{l}(i, \mathrm{j})$, and any image in the pyramid can be expanded $n$ times $(n>0$ and, usually, $n \leq l$, but this latter is not a necessary condition) in a stepwise manner, such that in each step the image is expanded to the size of the previous level in the pyramid by means of the following algorithm:

$$
\begin{aligned}
g_{l, n+1}(i, j)= & 4 \sum_{m, n=-2}^{2} \sum(m, n) \\
& \times g_{l, n}((i-m+1) / 2,(j-n+1) / 2),
\end{aligned}
$$

where $1 \leq i \leq R_{i-n-1}, 1 \leq j \leq C_{l-n-1}, R_{k}=M_{R} 2^{N-k}+1$, and $C_{k}=M_{C} 2^{N-k}+1$. In this sum, terms in which any of the array indexes are not integer-valued are taken to be zero (Burt, 1981).

Three different usages can be given to the images thus expanded. First, one may want to expand an image to 


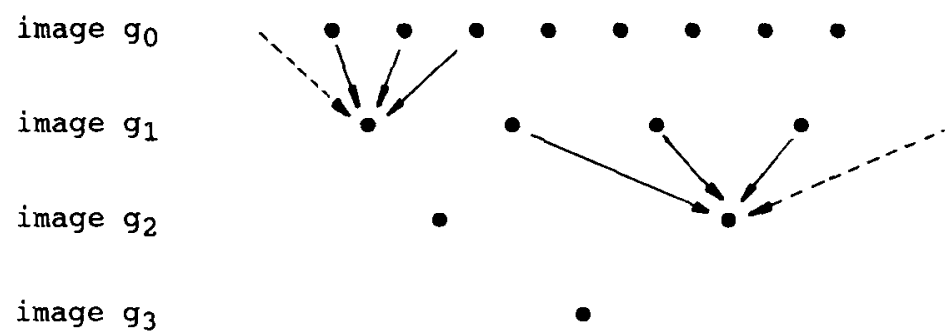

Figure 3. Illustration of the operation of the even-REDUCE function. If the image points at the output are taken for reference, the generating kernel does not have to be laterally displaced, because image points at the output fall midway between image points at the input.

make clearly visible what its contents are (see Burt \& Adelson, 1983a, top row of Figure 5). Second, in the context of pyramid operations, one may want to subtract the expanded image from the image in the corresponding level of the pyramid to get a bandpass image (see Burt \& Adelson, 1983a, bottom row of Figure 5). Third, one may want to add the expanded levels of a pyramid of bandpass images to recover the original image (see Burt \& Adelson, 1983a, Figure 9). All three possibilities have been included in this subroutine, whose calling sequence is

\section{CALL EXPOI2 (F,G,WK,NRF,NCF,NRG,NCG,AX,} SX,AY,SY,BT,OE)

where

F: two-dimensional array of dimensions NRF $\times$ NCF containing the input image to be expanded (INTEGER*2).

G: two-dimensional array of dimensions NRG $\times$ NCG that is to contain the output of the expansion (INTEGER $* 2$ ).

WK: work vector of at least length NRF $($ REAL*4). NRF: number of rows in array F (INTEGER*4).

NCF: number of columns in array F (INTEGER $* 4$ ).

NRG: number of rows in array $G$ [NRG $=2 * N R F-1]$ (INTEGER*4).

NCG: number of columns in array $\mathrm{G}[\mathrm{NCG}=2 * \mathrm{NCF}-1]$ (INTEGER*4).

$\mathrm{AX}, \mathrm{SX}, \mathrm{AY}, \mathrm{SY}$, and BT are the same as in the previous subroutines.

OE: choice for the output of the expansion (INTEGER*4):

$$
\begin{aligned}
& \mathrm{OE}<0 \longleftrightarrow \mathrm{G}=\mathrm{G}-\operatorname{EXPAND}(\mathrm{F}), \\
& \mathrm{OE}=0 \longleftrightarrow \mathrm{G}=\mathrm{EXPAND}(\mathrm{F}), \\
& \mathrm{OE}>0 \longleftrightarrow \mathrm{G}=\mathrm{G}+\operatorname{EXPAND}(\mathrm{F}) .
\end{aligned}
$$

Of course, the odd-EXPAND function can be applied to any image of appropriate dimensions out of the context of pyramid processing.

Subroutine EXPEI2 (EXPAND function, even form, integer arrays, $\hat{\boldsymbol{m}}=2$ ). As it happens with the odd and even forms of the REDUCE function, there is not any fundamental difference between the odd and even forms of the EXPAND function, except in what regards the size of the arrays involved in each case. The even-EXPAND algorithm is

$$
\begin{aligned}
g_{l, n+1}(i, j)= & 4 \sum_{m, n=-2}^{1} \sum_{l} w(m, n) \\
& \times g_{i, n}((i-m) / 2,(j-n) / 2),
\end{aligned}
$$

where $1 \leq i \leq R_{l-n-1}, 1 \leq j \leq C_{l-n-1}, R_{k}=M_{R} 2^{N-k}$, and $C_{k}=M_{c} 2^{N-k}$. The same considerations discussed with regard to the odd form of the algorithm can also be straightforwardly applied to this case. This subroutine can be called using the statement

\section{CALL EXPEI2 (F,G,WK,NRF,NCF,NRG,NCG,AX, SX,AY,SY,BT,OE)}

where the definitions and types of the arguments are the same as in the previous subroutine, except that now $\mathrm{NRG}=2 * \mathrm{NRF}$ and $\mathrm{NCG}=2 * \mathrm{NCF}$.

Figure 4 shows the Gaussian (left) and Laplacian (right) pyramid representations of a $256 \times 256$ image of the Roman aqueduct in Segovia. These images have been obtained recursively using subroutines REDEI2 and EXPEI2 with so simple a program as that in Appendix A. (Note that $\mathrm{AX}=\mathrm{AY}=0.375, \mathrm{SX}=\mathrm{SY}=1$, and $\mathrm{BT}=2$ in that program.)

The next eight subroutines are similar to the previous eight, but in these cases $\hat{m}=3$. The algorithms implemented are mere extensions of those in Equations 1-6 to cope with the larger widths of the kernels. Thus, the sums in Equations 1, 3, and 5 extend from -3 to 3; the sums in Equations 2.1, 4, and 6 extend from -3 to 2; and the sum in Equation 2.2 extends from -2 to 3 . Kernels with $\hat{m}=3$ are subjected to the same constraints as the smaller ones. For odd-width kernels, these constraints are satisfied (in one dimension) when

$$
\begin{aligned}
& w_{x}(0)=a_{x} \\
& w_{x}(1)=w_{x}(-1)=b_{x} \\
& w_{x}(2)=w_{x}(-2)=s_{x} / 4-a_{x} / 2 \\
& w_{x}(3)=w_{x}(-3)=s_{x} / 4-b_{x}
\end{aligned}
$$




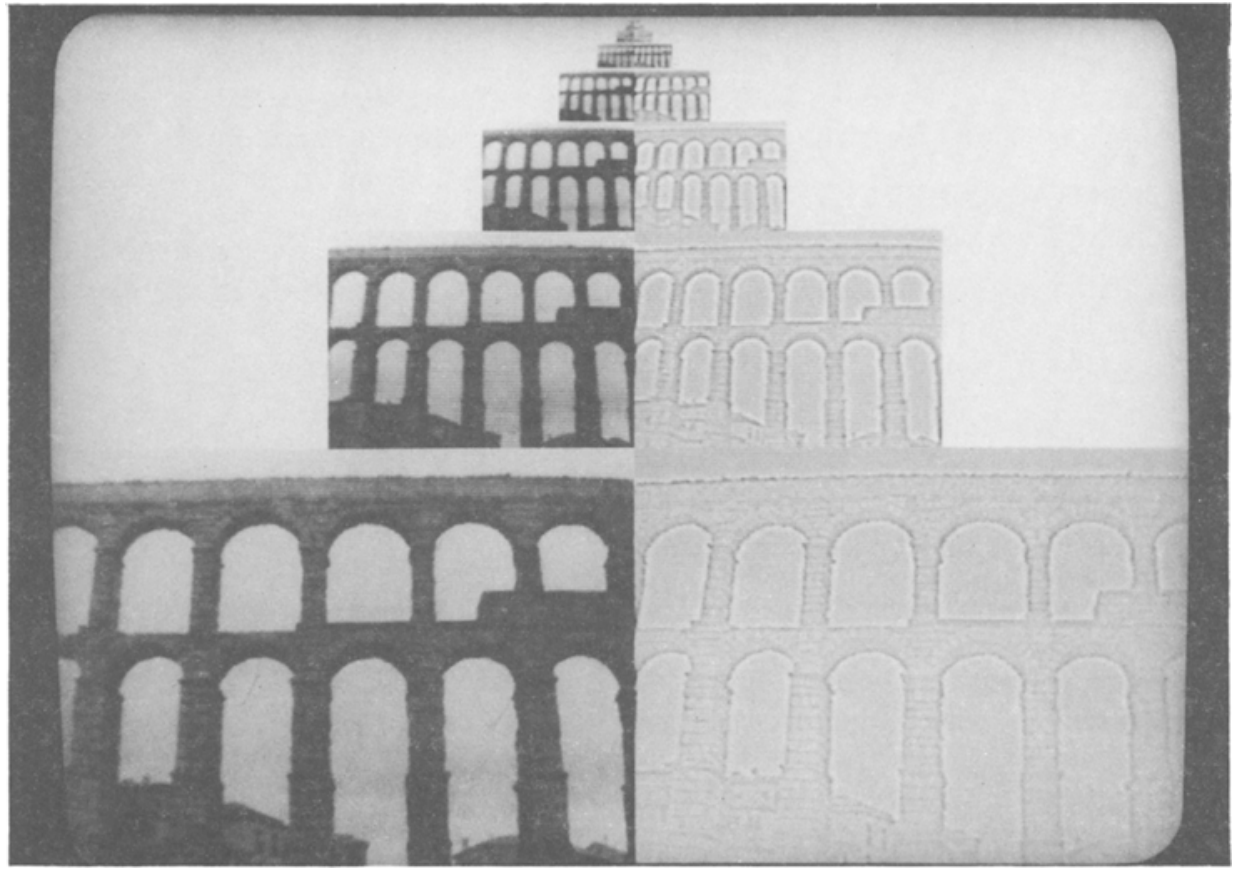

Figure 4. Gaussian (left) and Laplacian (right) pyramids constructed from a $256 \times 256$ image of the Roman aqueduct in Segovia. Each pyramid contains nine levels, although not all of them are visible in this photograph. The images in the Laplacian pyramid have been rescaled to avoid negative values and adjusted to fit in the 8-bit range of the graphic system.

and for even-width kernels of the type in the extension of Equation 2.1 when

$$
\begin{aligned}
& w_{x}(0)=w_{x}(-1)=a_{x} \\
& w_{x}(1)=w_{x}(-2)=b_{x} \\
& w_{x}(2)=w_{x}(-3)=s_{x} / 2-a_{x}-b_{x} .
\end{aligned}
$$

As can be seen, there are two free parameters in kernels of either even or odd widths for which $\hat{m}=3$, and, therefore, more complex convolution masks can be designed. we show later the use of this type of kernel for approximating Gabor filters similar to those used in current models of spatial vision (Daugman, 1984; Watson, 1983; Webster \& De Valois, 1985).

All the points raised with regard to the previous eight subroutines can also be applied directly to these eight. Therefore, only the calling sequence of each of these subroutines is described below.

Subroutines HDCOI3 and SMHOI3 (HDC algorithm, odd form, integer arrays, $\hat{m}=3$ ). Subroutine SMHOI 3 should be used when $C \leq 5$; otherwise, subroutine HDCOI3 should be used. These subroutines are called by using

CALL HDCOI3 (F,G,WK,NR,NC,AX,BX,SX,AY, $\mathrm{BY}, \mathrm{SY}, \mathrm{BT})$

and

CALL SMHOI3 (F,G,WK,NR,NC,AX,BX,SX,AY, $\mathrm{BY}, \mathrm{SY}, \mathrm{BT})$ where $\mathrm{BX}$ and $\mathrm{BY}$ are the second weight parameters of the generating kernel in the $x$ - and $y$-directions, respectively, and the rest of the arguments are as in subroutines HDCOI2 or SMHOI2.

Subroutines HDCEI3 and SMHEI3 (HDC algorithm, even form, integer arrays, $\hat{m}=3$ ). Subroutine HDCEI3 should be used if $C \geq 5$; otherwise subroutine SMHEI3 should be used. Their calling sequences are

$$
\begin{gathered}
\text { CALL HDCEI3 (F,G,WK,NR,NC,AX,BX,SX,AY, } \\
\text { BY,SY,BT,ALGX,ALGY) }
\end{gathered}
$$

and

\section{CALL SMHEI3 (F,G,WK,NR,NC,AX,BX,SX,AY,} BY,SY,BT,ALGX,ALGY)

where $\mathrm{BX}$ and $\mathrm{BY}$ are as in $\mathrm{HDCOI} 3$, and the rest of the arguments are as in subroutines HDCEI2 or SMHEI2.

Subroutine REDOI3 (REDUCE function, odd form, integer arrays, $\hat{m}=3$ ). The calling statement for this subroutine is

\section{CALL REDOI3 (F,G,WK,NRF,NCF,NRG,NCG, AX,BX,SX,AY,BY,SY,BT)}

where $\mathrm{BX}$ and $\mathrm{BY}$ are as in HDCOI3, and the rest of the arguments are as in subroutine REDOI2.

Subroutine REDEI3 (REDUCE function, even form, integer arrays, $\hat{\boldsymbol{m}}=\mathbf{3}$ ). This subroutine is called by using

CALL REDEI3 (F,G,WK,NRF,NCF,NRG,NCG, AX,BX,SX,AY,BY,SY,BT) 
where BX and BY are as in the previous subroutine, and the rest of the arguments are as in subroutine REDEI2.

Subroutine EXPOI3 (EXPAND function, odd form, integer arrays, $\hat{m}=3$ ). Its calling sequence is

\section{CALL EXPOI3 (F,G,WK,NRF,NCF,NRG,NCG, AX,BX,SX,AY,BY,SY,BT,OE)}

where $\mathrm{BX}$ and $\mathrm{BY}$ are as in the previous subroutine, and the rest of the arguments are as in subroutine EXPOI2.

Subroutine EXPEI3 (EXPAND function, even form, integer arrays, $\hat{m}=\mathbf{3}$ ). The calling sequence for this subroutine is

\section{CALL EXPEI3 (F,G,WK,NRF,NCF,NRG,NCG, AX,BX,SX,AY,BY,SY,BT,OE)}

where BX and BY are as in the previous subroutine, and the rest of the arguments are as in subroutine EXPEI2.

As stated before, Gabor filters can be easily approximated by means of kernels of width 7 . The program in Appendix B has been used to obtain the images in Figure 5, which shows a Gabor pyramid of the Roman aqueduct in Segovia. This pyramid is constructed by applying subroutine HDCOI3 to each of the images in the Gaussian pyramid shown in the left half of Figure 4. (Note that, in that program, $\mathrm{AX}=0.5, \mathrm{BX}=-0.1, \mathrm{SX}=0$, $\mathrm{AY}=0.338, \mathrm{BY}=0.237, \mathrm{SY}=1$, and $\mathrm{BT}=2$.) The operation of a bank of Gabor filters of equal bandwidth and differing in center frequency is efficiently approximated by applying the same small-size kernel with suitably chosen weights to each of a set of subsampled, band- limited images. There is nothing wrong in applying the Gabor kernel to drastically lowpass filtered images (such as those in high levels of the Gaussian pyramid), provided they have been constructed with appropriate subsampling. Figure 6 shows the first four images in the Gabor pyramid after they have been expanded to the size of the original image using Gaussian interpolation. (The program segment that does this work is also shown in Appendix B.)

\section{Computational Efficiency}

As discussed by Burt (1981), the HDC is in itself more efficient than either direct convolution or convolution via FFT. The virtue of the implementation described in this paper is that it reduces the number of additions and multiplications in the original algorithms by taking full advantage of the separability of the two-dimensional generating kernel and of the symmetry in the one-dimensional kernels. Also, 16 different, although in some cases very similar, subroutines have been written to avoid branching and unnecessary operations within a single, general subroutine, and the code in each one has been carefully optimized to minimize the number of operations other than the required additions and multiplications. The result of this work is a set of subroutines whose computational efficiency is greater than that that would have resulted had Equations 1-6 and their extensions to larger kernel widths been directly implemented. To give an idea of this improved efficiency, Table 1 lists the execution times of each subroutine for several input image sizes when the executive programs run on an Olivetti M24. Note that, under

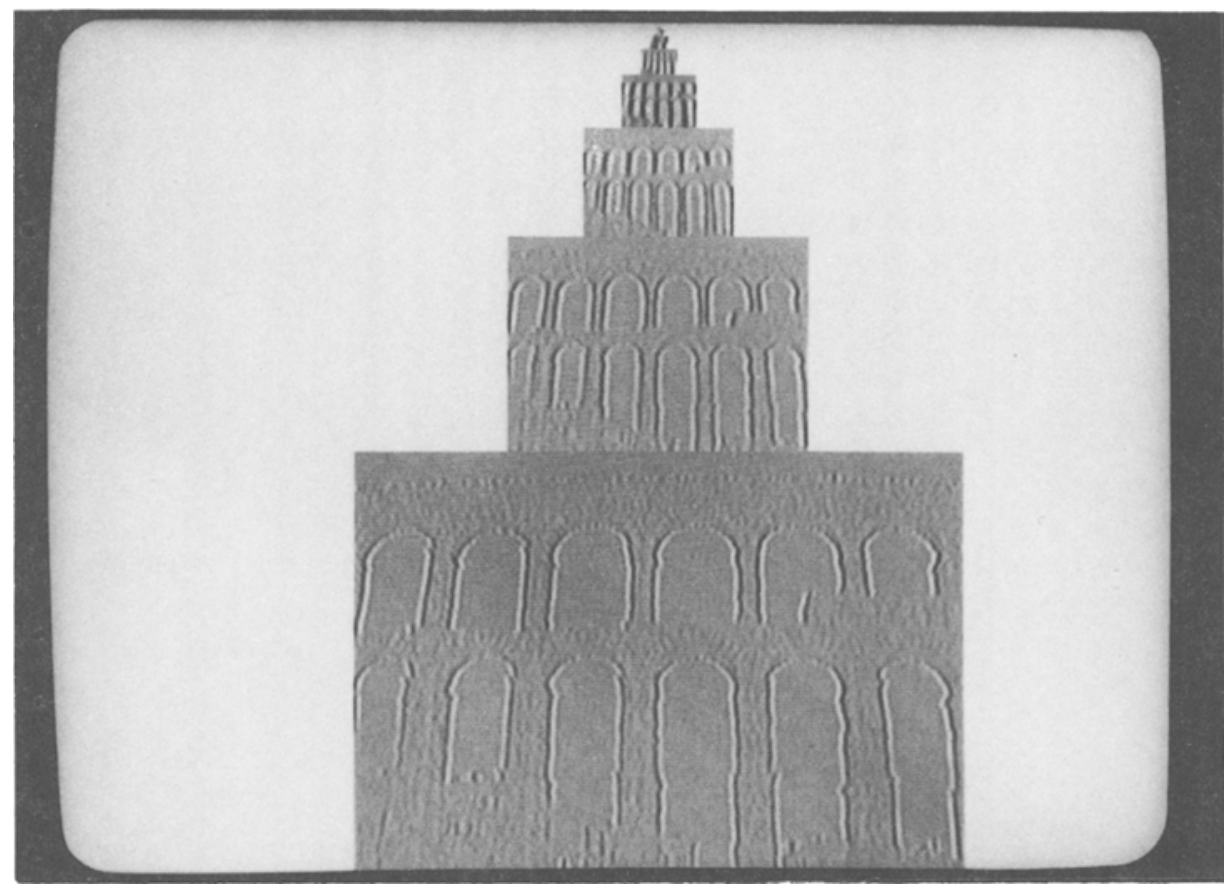

Figure 5. Gabor pyramid constructed from the Gaussian pyramid in Figure 4. Images have been rescaled and adjusted to fit in the 8-bit range of the graphic system. 


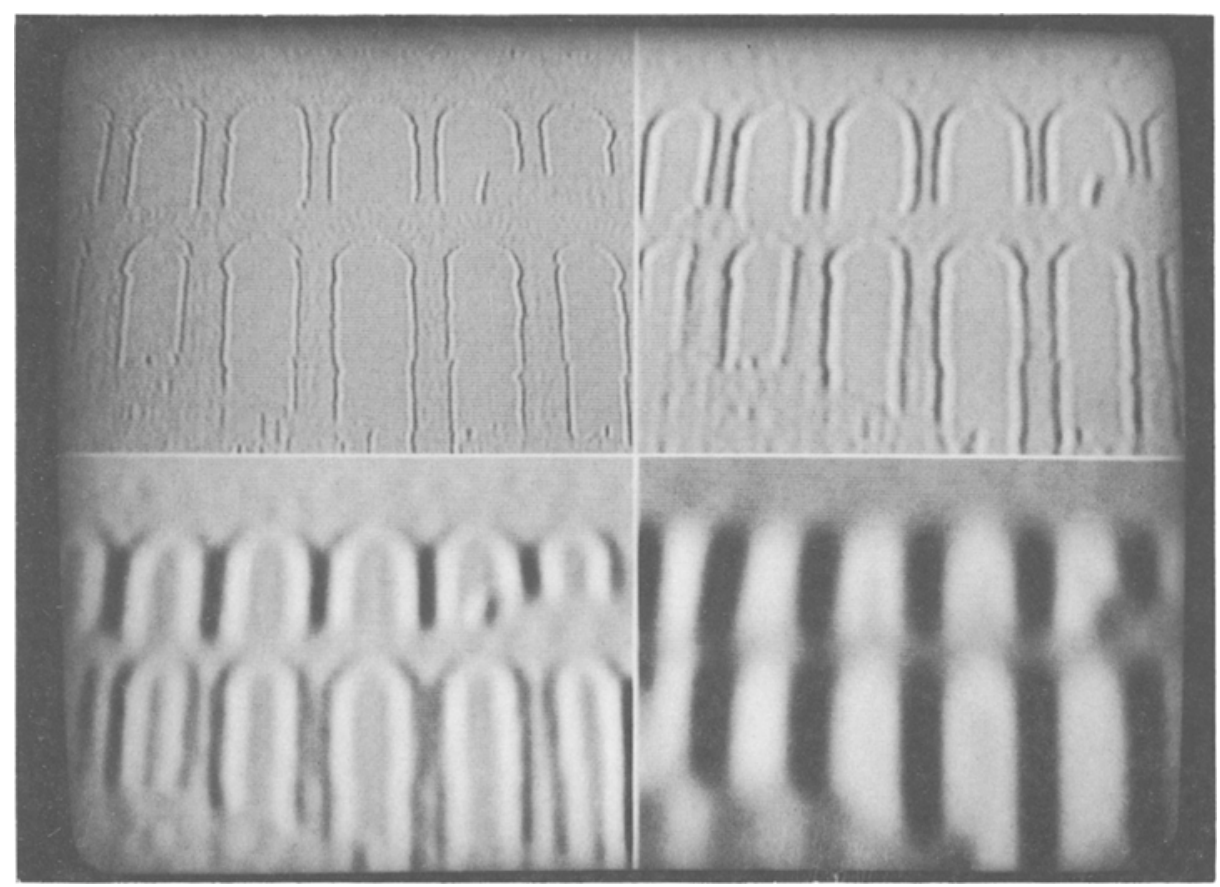

Figure 6. First four levels of the Gabor pyramid shown in Figure 5, expanded to the size of the original image to make their contents clearly visible. Top left, level 0 ; top right, level 1; bottom left, level 2, bottom right, level 3.

our implementation, convolutions with large kernels require roughly $33 \%$ more time than convolutions with smaller kernels, even though $7 \times 7$ or $6 \times 6$ neighborhoods have about $100 \%$ more pixels than $5 \times 5$ or $4 \times 4$ neighborhoods (compare the execution time of any $\hat{m}=3$ subroutine with that for the corresponding $\hat{m}=2$ subroutine). All of these times have been estimated using the Professional FORTRAN subroutine GETTIM (Ryan-McFarland, 1984). The execution time is not significantly affected by the method selected for treatment of boundaries; for subroutines EXPOI2, EXPO13, EXPEI2, and EXPEI3, the execution time depends slightly on the choice for the output of the expansion (argument $\mathrm{OE}$ ), and the values reported in Table 1 are mean times. Nevertheless, the differences between options vary within $\pm 5 \%$ of the time reported for each image size, the fastest execution corresponding to $\mathrm{OE}=0$ and the slowest to $\mathrm{OE}<0$, which requires an additional subtraction per image point. For comparison, a direct implementation of Equation 1 takes $487 \mathrm{sec}$ when the input image is $257 \times 257$.

\section{Conclusions}

We have presented a library of subroutines that efficiently perform HDC calculations. Algorithms for full-density HDC, as well as for REDUCE and EXPAND operations, have been included in both their even and odd forms. In each case, algorithms for odd kernels of widths $5 \times 5$ and $7 \times 7$ and even kernels of widths $4 \times 4$ and $6 \times 6$ have been implemented. This by no means implies that other kernel widths within these limits cannot be programmed with these subroutines. In fact, as a consequence of the constraints on the generating kernel, small kernels are degenerate cases of large kernels. Therefore, the $3 \times 3$ kernel used by Burton et al. (1986) can be obtained with subroutine REDOI 2 by setting $A X=A Y=0.5$ and $S X=S Y=1$. The reason why specific subroutines for kernels of width $3 \times 3$ (or $2 \times 2$ in the even form) have not been written is that these smallest kernels have no free parameters, and, therefore, they can give rise only to a single filter shape, which is scarcely used in either visual modeling or image processing. If, however, this kind of

Table 1

Execution Time (in sec) of Each Subroutine for Several Image Sizes

\begin{tabular}{lccr}
\hline & \multicolumn{3}{c}{ Input Image Size } \\
\cline { 2 - 4 } Subroutine & $257 \times 257$ & $129 \times 129$ & $65 \times 65$ \\
\hline HDCOI2 & 65.4 & 16.6 & 4.2 \\
HDCOI3 & 86.9 & 21.9 & 5.6 \\
REDOI2 & 26.2 & 6.6 & 1.7 \\
REDO13 & 34.4 & 8.7 & 2.2 \\
EXPOI2 & & 34.1 & 8.6 \\
EXPOI3 & & 41.5 & 10.5 \\
& & $128 \times 128$ & $64 \times 64$ \\
\hline HDCEI2 & 57.4 & 14.4 & 3.6 \\
HDCEI3 & 78.4 & 19.6 & 4.9 \\
REDEI2 & 20.8 & 5.2 & 1.3 \\
REDEI3 & 28.8 & 7.2 & 1.8 \\
EXPEI2 & & 34.0 & 8.5 \\
EXPEI3 & & 41.5 & 10.4 \\
\hline
\end{tabular}


kernels were needed either for practical or for experimental purposes, they can be implemented following the above-mentioned procedure.

Similar considerations apply to the design of nonsquare kernels, such as $7 \times 3$ or $4 \times 6$. The former can be programmed by setting AY, BY, and SY as desired (but not $\mathrm{BY}=\mathrm{SY} / 4$, or simultaneously $\mathrm{AY}=\mathrm{SY} / 2$ and $\mathrm{BY}=$ $S Y / 4)$ and $A X=S X / 2$ and $B X=S X / 4$, and the latter by setting $A X, B X, S X, A Y$, and $S Y$ as desired (but not $\mathrm{BX}=\mathrm{SX} / 2-\mathrm{AX}$ ) and $\mathrm{BY}=\mathrm{SY} / 2-\mathrm{AY}$. Likewise, any other combination can be easily programmed, taking into consideration the relationships between inner and outer parameters in the generating kernel.

To avoid a relatively high presence of border effects, it is required that the image be at least $2 \hat{m}$ pixels wider than the kernel. However, this point is left up to the user's consideration, and all subroutines have been prepared to handle images of even the smallest size within the theoretical limits of the algorithm implemented, regardless of the width of the kernel. Thus, HDC subroutines can handle images as small as $1 \times 1$ pixels; subroutines REDOI2 and REDOI3 require that the horizontal and vertical widths of the input image be odd and not fewer than 3 pixels for the smallest dimension; subroutines REDEI2 and REDEI3 merely require that the input image have an even number of pixels per side (as few as 2 pixels for the smallest dimension is allowed); subroutines EXPOI2 and EXPOI3 require that the widths of the input image be greater than or equal to 2 pixels for the smallest dimension (recall that the output image will always have odd widths, regardless of whether the widths of the input image are even or odd); subroutines EXPEI2 and EXPEI3 can handle images of any size (even a single pixel for the smallest dimension), but recall that the output image will have even widths, regardless of the widths of the input image.

For filters without circular symmetry, it is sometimes convenient to apply the filter at each of several orientations. This is particularly useful when dealing with Gabor filters for modeling human vision. However, with small, constrained kernels on a square grid, it is difficult to implement filters at orientations other than horizontal or vertical. Filters at the two oblique orientations can, in fact, be implemented using HDC of fractional order (see Burt, 1981), but the large width of the Gabor kernel complicates the algorithm and it makes its implementation hardly efficient. We propose, instead, to first rotate the input image the desired angle, then apply the filter in a standard vertical or horizontal orientation, and finally rotate the output image by the same angle but in the opposite direction. Rotating the image would be trivial if it could be done by rotating the grid, but that is not possible. Then image features can be rotated instead by displacing the image values through the square grid of the array in such a way that the relative positions of each pair of values in the original image remain unchanged in the rotated image and, also, that the image values in a given row (or column) in the original image occupy the grid positions that are closer to a line at the selected orientation in the rotated image.

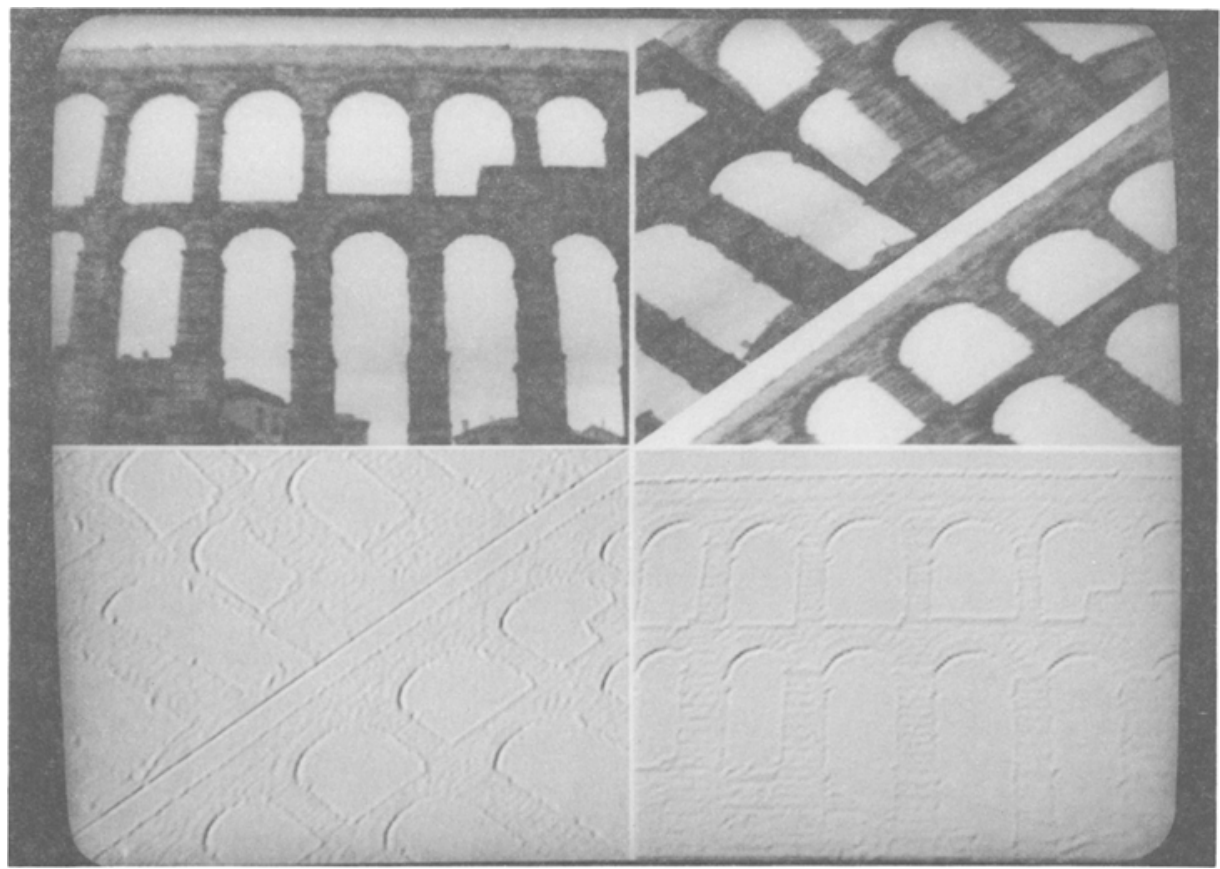

Figure 7. Hlustration of the method for filtering at a given orientation band when using orientationselective kernels. Top left, original image; top right, $45^{\circ}$ counterclockwise rotated image; bottom left, result of applying the Gabor kernel to the rotated image; bottom right, filtered image after reversing the rotation. See the text for details. 
This method is illustrated in Figure 7 (top). The original image is shown on the left, and the rotated image appears on the right. The rotation involves two steps. In the first step, the two first (upper) rows of the image are left unchanged, and each subsequent pair of rows is displaced to the right a distance 1 pixel larger than the displacement applied to the immediately preceding pair. Leading grid points at the left of each row are filled in with the image values at the right, which fall out of the grid due to the displacement of the row (i.e., the left and right image edges are regarded as adjacent). In the second step, the first (leftmost) column is not displaced, and each subsequent column is displaced upward a distance 1 pixel larger than the displacement applied to the immediately preceding column. Leading grid points are filled in using an analogous procedure to that used for rows.

This is the closest we can get to a $45^{\circ}$ counterclockwise rotation while keeping invariant the square grid of the image. Rotations at different angles or directions can be accomplished easily by varying the direction of displacement of rows and columns, the sizes of the blocks of rows and columns that are displaced by the same amount, and the magnitude of the increment in displacement from one block to the next. Note that different parts of the original image are copied to different positions in the rotated image, so that the orientation of all image features changes appropriately. Since the HDC involves only local operations, the topological distortion in the rotated images is not critical for the results. We can now use subroutine HDCOI3 to apply a vertically oriented Gabor kernel to the rotated image in order to produce the image shown in the bottom left of Figure 7. It is important to note that we must use rule $b$ for boundary treatment (i.e., $\mathrm{BT}=1$ ), since the actual image values beside each edge of the rotated image are those at the opposite edge, as can be seen by inspection of the image in the top right of Figure 7. This is also the rule that has been forced for actual image edges when rotating the image, as can be seen in the off diagonal of the rotated image and the first half of the main diagonal. ${ }^{3}$ Finally, reversing the rotation on the filtered image gives rise to the image in the bottom right of Figure 7. Obviously, this image looks as if it had been filtered using a Gabor kernel rotated clockwise by $45^{\circ}$. Nonetheless, the filtered image thus obtained is only an approximation to the desired result. Figure $8 b$ shows the shape and distribution of weights of a kernel, which, if applied to the original image, would have produced the same result as the kernel in Figure 8a when applied to the rotated image. As can be seen, the kernel in Figure $8 \mathrm{~b}$ does not exactly correspond to a $45^{\circ}$ rotation of the kernel in Figure 8a, but this is the closest approximation.

For the sake of computational efficiency, arguments are not checked for incorrect values by these subroutines, and it is therefore extremely important that the CALL statement be carefully written, especially with respect to the arguments regarding options (BT, ALGX, and ALGY) or those concerning output array dimensions in the REDUCE and EXPAND subroutines, as well as in the correct use of HDC* versus SMH* subroutines.

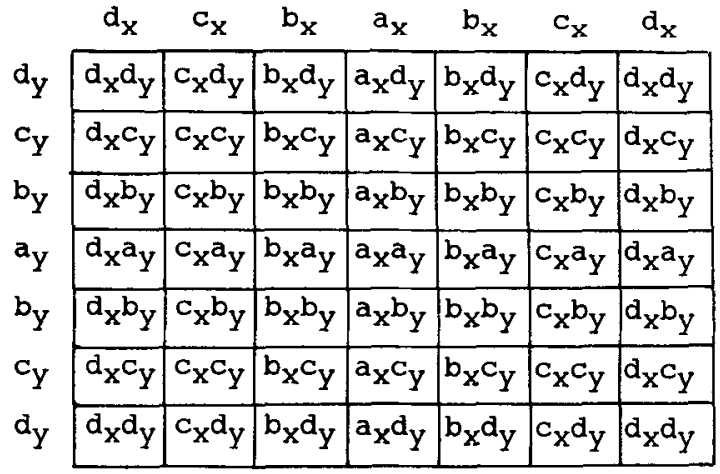

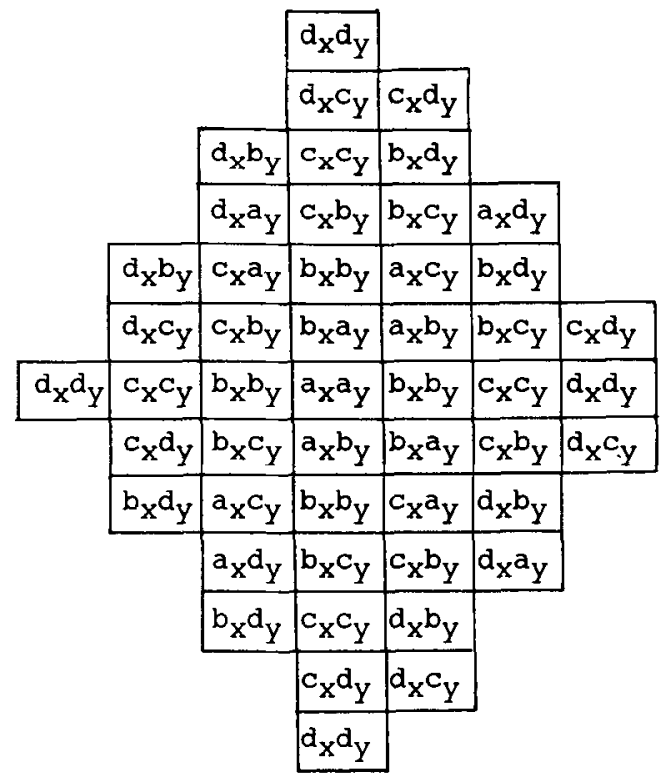

Figure 8. (a) Shape and distribution of weights of an odd-width kernel with $\hat{m}=3$. (b) Shape and distribution of weights of the equivalent kernel at the original image when the kernel in (a) is applied to an image that has undergone the rotation described in the text. These features obviously depend on the magnitude and direction of rotation applied to the original image. 


\section{Availability}

The source code for the library is not available at present. The library of object modules can be obtained at a cost of 15,000 pesetas. Contact the author for additional information.

\section{REFERENCES}

Arbeiter, J. H., \& Bessler, R. F. (1986). A two-dimensional realtime video pyramid processor. RCA Review, 47, 3-31.

BRACEWELL, R. N. (1978). The Fourier transform and its applications. New York: McGraw-Hill.

Burt, P. J. (1981). Fast filter transforms for image processing. Computer Graphics \& Image Processing, 16, 20-51.

BURT, P. J. (1983). Fast algorithms for estimating local image properties. Computer Vision, Graphics, \& Image Processing, 21, 368-382.

Burt, P. J., \& Adelson, E. H. (1983a). The Laplacian pyramid as a compact image code. IEEE Transactions on Communications, COM$31,532-540$.

BurT, P. J., \& ADELson, E. H. (1983b). A multiresolution spline with application to image mosaics. ACM Transactions on Graphics, 2, 217-236.

Burton, G. J., Haig, N. D., \& Moorhead, I. R. (1986). A self-similar stack model for human and machine vision. Biological Cybernetics, 53, 397-403.

Crowley, J. L., \& STERN, R. M. (1984). Fast computation of the difference of low-pass transform. IEEE Transactions on Pattern Analysis and Machine Intelligence, PAMI-6, 212-222.

Daugman, J. G. (1984). Spatial visual channels in the Fourier plane. Vision Research, 24, 891-910.

DuhameL, P. (1986). Implementation of "split-radix" FFT algorithms for complex, real, and real-symmetric data. IEEE Transactions on Acoustics, Speech, \& Signal Processing, ASSP-34, 285-295.

García-PÉrez, M. A. (1986). An efficient approach to modeling in- homogeneity within the visual system. Manuscript submitted for publication.

Graham, N., Kramer, P., \& Haber, N. (1985). Attending to the spatial frequency and spatial position of near-threshold visual patterns. In M. I. Posner \& O. S. M. Marin (Eds.), Attention and performance XI (pp. 269-284). Hillsdale, NJ: Erlbaum.

Richards, W., Dawson, B., \& Whittington, D. (1986). Encoding contour shape by curvature extrema. Journal of the Optical Society of America, A3, 1483-1491.

Ryan-McFarland Corporation (1984). IBM Personal Computer Professional FORTRAN reference manual.

WATSON, A. B. (1983). Detection and recognition of simple spatial forms. In O. J. Braddick \& A. C. Sleigh (Eds.), Physical and biological processing of images (pp. 100-114). Berlin: Springer-Verlag.

WATSON, A. B., \& AHUMADA, A. J., JR. (1985). Model of human visualmotion sensing. Joumal of the Optical Sociery of America, A2, 322-342.

Webster, M. A., \& DE Valois, R. L. (1985). Relationship between spatial-frequency and orientation tunning of striate-cortex cells. Journal of the Optical Society of America, A2, 1124-1132.

\section{NOTES}

1. For the sake of simplicity, throughout the paper the horizontal and vertical widths of the kernels are considered equal. Elongated kernels, such as $7 \times 5$ or $2 \times 6$, are discussed in the Conclusions section.

2. In actual fact, the library contains 28 subroutines, but 12 are lower level subroutines of the 16 subroutines described here.

3. There is, however, an unavoidable mismatch between lower and upper edges in the original image along the off diagonal in the rotated image. Note that the left half of the bottom edge in the original image is copied beside the right half of the top edge and, of course, the right half of the bottom edge in the original image appears beside the left half of the top edge. This misalignment will only affect, although quite insignificantly, the filtering performed on the five rows of pixels nearest to either bottom or top edges when the rotation is reversed.

\author{
APPENDDX A \\ Main Structure of Program Used to Construct Pyramids Shown in Figure 4

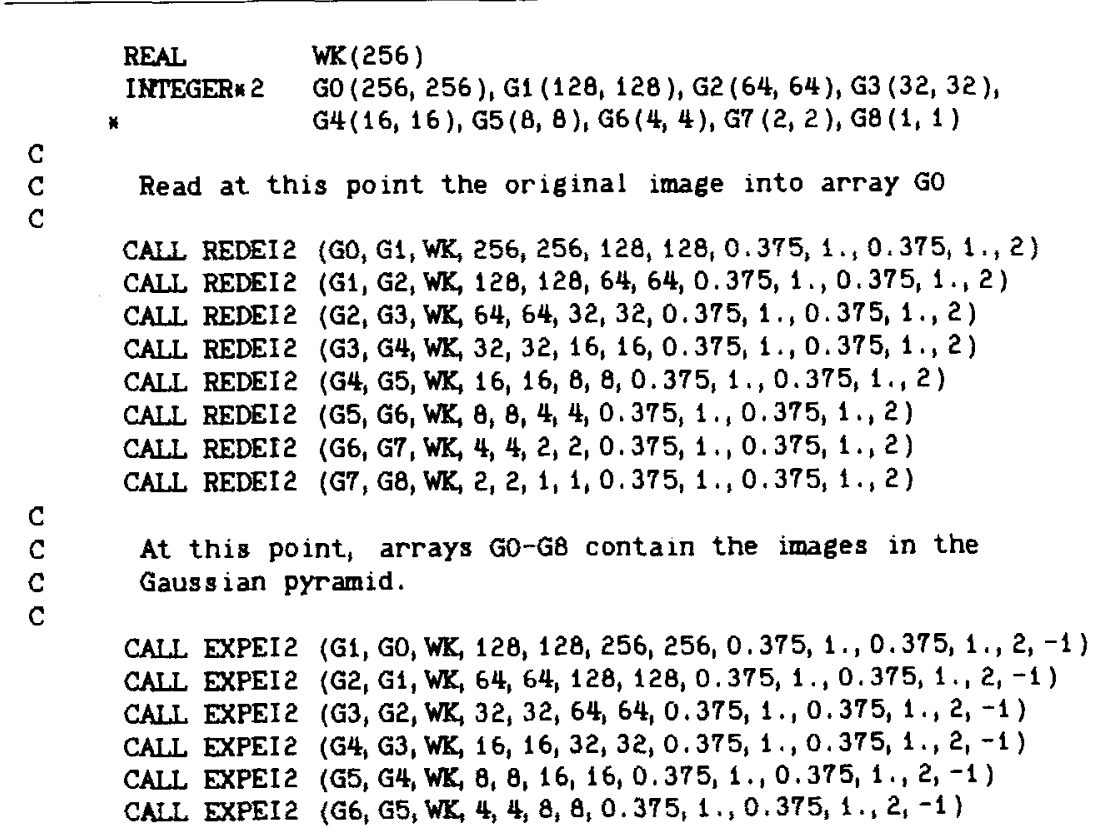




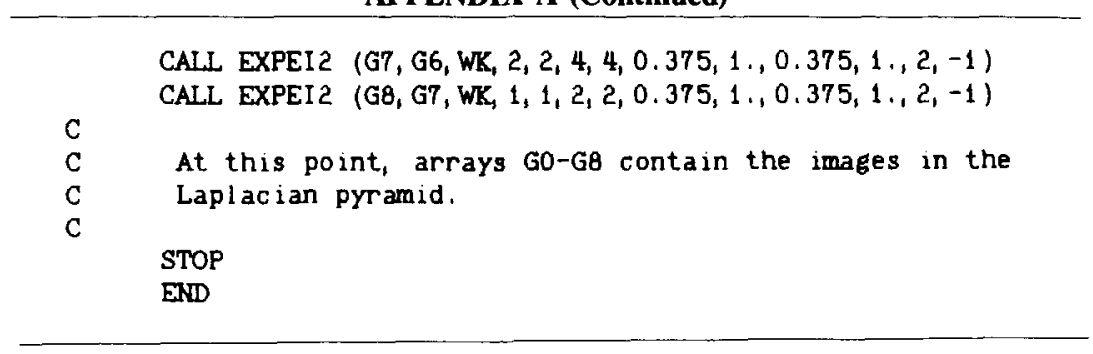

APPENDIX B

Main Structure of Program Used to Create Images Shown in Figures 5 and 6

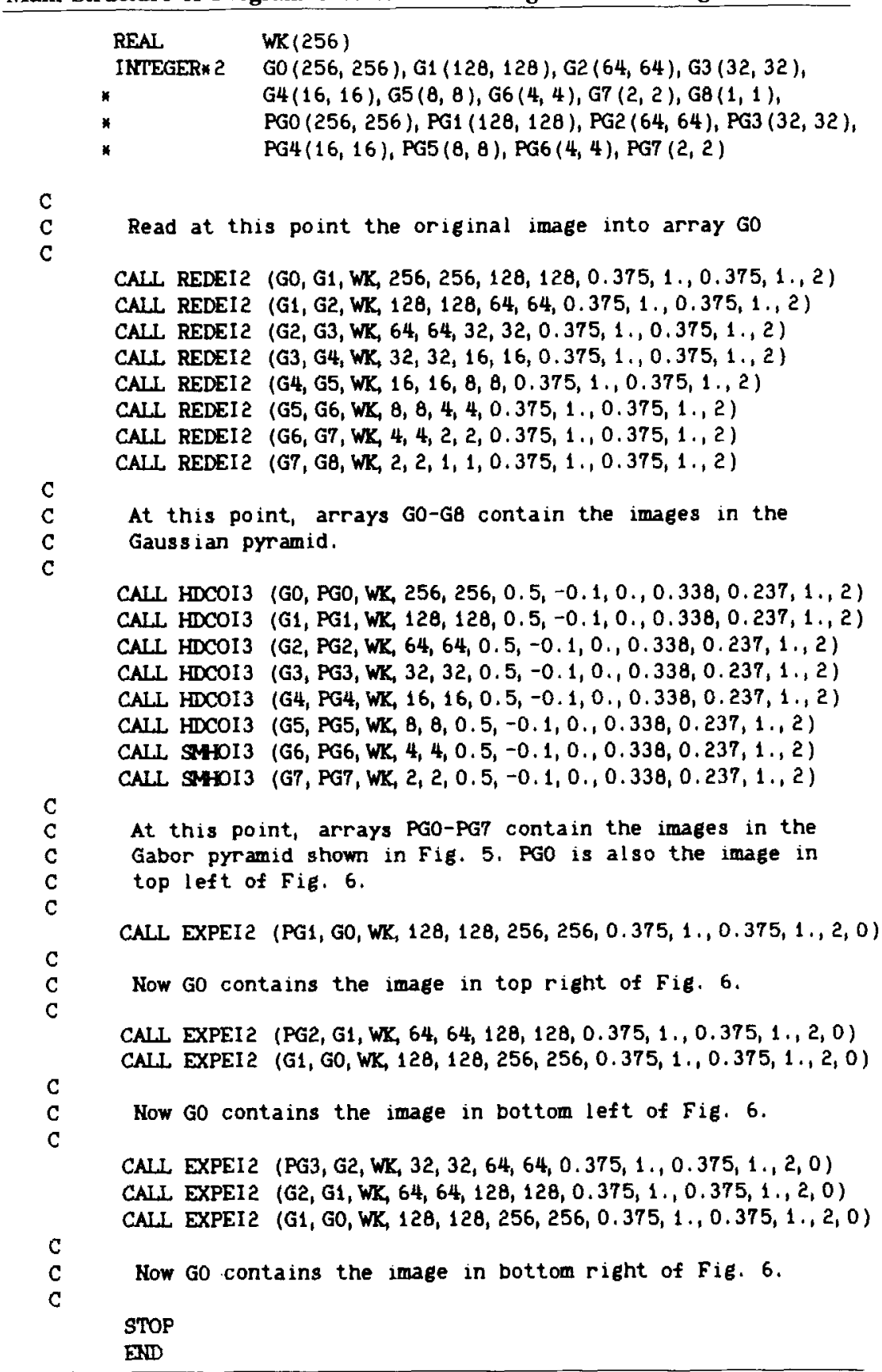

